\title{
Hh-1 Antigen
}

National Cancer Institute

\section{Source}

National Cancer Institute. Hh-1 Antigen. NCI Thesaurus. Code C17496.

the products of "hybrid histocompatibility" (Hh) loci, responsible for the phenomenon known as hybrid resistance. (from Fundamental Immunology, 3rd ed., William Paul, ed., Raven Press, NY 1993, pg 1104-1105) 